\title{
An open-label study of algorithm-based treatment versus treatment-as-usual for patients with schizophrenia
}

This article was published in the following Dove Press journal:

Neuropsychiatric Disease and Treatment

10 October 2013

Number of times this article has been viewed

\author{
Jinichi Hirano ${ }^{1,2}$ \\ Koichiro Watanabe ${ }^{3}$ \\ Takefumi Suzuki ${ }^{1,4}$ \\ Hiroyuki Uchida' \\ Ryosuke Den ${ }^{5}$ \\ Taishiro Kishimoto' \\ Takashi Nagasawa ${ }^{5}$ \\ Yusuke Tomita ${ }^{4}$ \\ Koichiro $\mathrm{Hara}^{6}$ \\ Hiromi Ochi ${ }^{7}$ \\ Yoshimi Kobayashi' \\ Mutsuko Ishii \\ Akane Fujita' \\ Yoshihiko Kanai' \\ Megumi Goto' \\ Hiromi Hayashi' \\ Kanako Inamura' \\ Fumiko Ooshima \\ Mariko Sumida' \\ Tomoko Ozawa' \\ Kayoko Sekigawa' \\ Maki Nagaoka' \\ Kae Yoshimura' \\ Mika Konishi' \\ Ataru Inagaki \\ Takuya Saito ${ }^{8}$ \\ Nobutaka Motohashi ${ }^{9}$ \\ Masaru Mimura' \\ Yoshiro Okubo ${ }^{8}$ \\ Motoichiro Kato \\ 'Department of Neuropsychiatry, Keio \\ University School of Medicine, Tokyo, \\ Japan; ${ }^{2}$ Ohizumi Hospital, Tokyo, Japan; \\ ${ }^{3}$ Department of Psychiatry School of \\ Medicine, Kyorin University, Tokyo, \\ Japan; ${ }^{4}$ Inokashira Hospital, Tokyo, Japan \\ ${ }^{5}$ Komagino Hospital, Tokyo, Japan; ${ }^{6}$ Asai \\ Hospital, Chiba, Japan; ${ }^{7}$ Kurumegaoka \\ Hospital, Tokyo, Japan; ${ }^{8}$ Department of \\ Psychiatry School of Medicine, Nippon \\ Medical School, Tokyo, Japan; 'Department \\ of Psychiatry, School of Medicine, \\ University of Yamanashi, Yamanashi, Japan
}

Correspondence: Jinichi Hirano

Keio University School of Medicine,

Department of Neuropsychiatry,

35, Shinanomachi, Shinjuku-ku,

Tokyo, 160-8582, Japan

Tel +81353633829

Fax +81353790187

Email hjinichi@aurora.ocn.ne.jp
Objective: The use of an algorithm may facilitate measurement-based treatment and result in more rational therapy. We conducted a 1-year, open-label study to compare various outcomes of algorithm-based treatment (ALGO) for schizophrenia versus treatment-as-usual (TAU), for which evidence has been very scarce.

Methods: In ALGO, patients with schizophrenia (Diagnostic and Statistical Manual of Mental Disorders, fourth edition) were treated with an algorithm consisting of a series of antipsychotic monotherapies that was guided by the total scores in the positive and negative syndrome scale (PANSS). When posttreatment PANSS total scores were above $70 \%$ of those at baseline in the first and second stages, or above $80 \%$ in the 3rd stage, patients proceeded to the next treatment stage with different antipsychotics. In contrast, TAU represented the best clinical judgment by treating psychiatrists.

Results: Forty-two patients ( 21 females, $39.0 \pm 10.9$ years-old) participated in this study. The baseline PANSS total score indicated the presence of severe psychopathology and was significantly higher in the ALGO group $(n=25 ; 106.9 \pm 20.0)$ than in the TAU group $(n=17$; $92.2 \pm 18.3)(P=0.021)$. As a result of treatment, there were no significant differences in the PANSS reduction rates, premature attrition rates, as well as in a variety of other clinical measures between the groups. Despite an effort to make each group unique in pharmacologic treatment, it was found that pharmacotherapy in the TAU group eventually became similar in quality to that of the ALGO group.

Conclusion: While the results need to be carefully interpreted in light of a hard-to-distinguish treatment manner between the two groups and more studies are necessary, algorithm-based antipsychotic treatments for schizophrenia compared well to treatment-as-usual in this study.

Keywords: algorithm, open-label clinical trial, measurement-based treatment, schizophrenia, treatment-as-usual

\section{Introduction}

Measurement-based treatment is of high importance in every field of medicine including psychiatry, ${ }^{1}$ and the process may be facilitated by the implementation of treatment recommendations and algorithms. However, there has been little evidence on treatments that are guided by an algorithm for psychotic disorders. In fact, a MEDLINE search (March 2013) could identify only two clinical trials that compared algorithm-based treatment (ALGO) with treatment-as-usual (TAU) in patients with schizophrenia., ${ }^{2,3}$ Furthermore, such a lack of clinical trials using ALGO appears to be the case for other psychiatric illnesses as well, including major depressive disorders. ${ }^{4}$

Previously, the Texas Medication Algorithm Project found a significant decrease in the Brief Psychiatric Rating Scale (BPRS) scores during the first 3 months of ALGO 
when compared with the TAU group. ${ }^{2}$ Over the subsequent 9 months, however, the TAU group demonstrated a significant decline in symptoms, eventually catching up with the ALGO group. Further, this study could not find significant differences in terms of quality of life, depression, and cognition, although ALGO patients had more frequent medication changes and a greater number of medication visits, especially in the early treatment stage.

Janssen et $\mathrm{al}^{3}$ compared the effectiveness of a computerbased, guideline-oriented, decision-support system with TAU. The study could not find a significant effect in the Positive and Negative Syndrome Scale (PANSS) total scores at any time points during the 12-month treatment, although ALGO was favorable in terms of rehospitalization. As such, the authors concluded that an ALGO was feasible and effective.

However, these clinical trials generally targeted relatively stable outpatients. Indeed, the average BPRS score was 38.8 to 45.4 in the Texas Medication Algorithm Project. ${ }^{2}$ The average score in the positive subscale of the PANSS was 13.8 to 14.5, and that for the negative subscale was 17.9 to 20.5 in the study by Janssen et $\mathrm{al}^{3}$ (total PANSS score not provided), thereby leaving the usefulness of the ALGO unaddressed in more severe patient populations. Additionally, such very limited evidence on systematic measurement-based treatments in schizophrenia originated from the US and Germany, and further evidence from the rest of the globe is critical. In Japan, pharmacotherapy of schizophrenia has been notoriously characterized with high-dose antipsychotic polypharmacy. ${ }^{5,6}$ The successful implementation of a treatment algorithm is expected to counteract antipsychotic polypharmacy and facilitate monotherapy - the latter of which has been endorsed in the treatment of schizophrenia. ${ }^{7}$

Based on such limited information, we report on the results from an open-label study in which a measurementbased treatment algorithm was adopted in the treatment of schizophrenia for the first time from Japan.

\section{Methods}

\section{Inclusion criteria}

Patients with schizophrenia, according to the Diagnostic and Statistical Manual of Mental Disorders, fourth edition, ${ }^{8}$ were recruited. To be eligible, the patients were required to be 20 to 65 years old and show a score range of 11 to 90 in the Global Assessment of Functioning (GAF), ${ }^{8}$ as well as a score of 3 (mild) to 6 (severe) in the Clinical Global ImpressionSchizophrenia (CGI-SCH) overall severity subscale. ${ }^{9}$ Also, all patients were required to have received antipsychotic treatment at chlorpromazine-equivalent doses of $150 \mathrm{mg}$ to $2000 \mathrm{mg}$ per day. ${ }^{10}$

\section{Exclusion criteria}

Exclusion criteria included: a clear history of nonresponse or intolerance to any of the atypical antipsychotics (ie, olanzapine, risperidone, quetiapine. aripiprazole, perospirone, or blonanserin); active and uncontrolled somatic conditions; significant personality disorders or mental retardation severe enough to interfere with the ability to give consent; a potential of being pregnant; a significant risk of being suicidal; and active substance abuse.

\section{Trial setting}

This was a prospective, multisite, open-label study of ALGO in Japan. This clinical trial was conducted at eight hospitals (Keio University, Nippon Medical School, University of Yamanashi, Ohizumi Hospital, Inokashira Hosipital, Komagino Hospital, Asai Hospital, Kurumegaoka Hospital). The study was approved by the institutional review board at each participating site, and written informed consent was obtained from all patients after a full description of the study. This study received financial support from the Japanese Minister of Health, Labour, and Welfare. Data were collected from July 2009 through June 2012.

\section{Outcome measurements}

The primary outcome measure was a total score on the PANSS. ${ }^{11}$ The secondary outcome measures aimed to address a variety of aspects in the illness and included the CGI-SCH, ${ }^{9}$ GAF, ${ }^{8}$ Drug-Induced Extra-Pyramidal Symptoms Scale (DIEPSS), ${ }^{12}$ Targeted Inventory on Problems in Schizophrenia (TIP-Sz), ${ }^{13}$ Functional Assessment for Comprehensive Treatment of Schizophrenia (FACT-Sz), ${ }^{13}$ Medical Outcome Survey Short-form 36v2 Health Survey (SF-36v2), ${ }^{14}$ Japanese version of Subjective Well-Being under Neuroleptics Scale (SWN-J), ${ }^{15}$ and Drug Attitude Inventory (DAI-10). ${ }^{16}$

The duration of the study was 52 weeks. All patients were evaluated every 4 weeks with the PANSS, CGI-SCH, GAF, DIEPSS, TIP-Sz, FACT-Sz, SF-36v2, SWN-J, and DAI10. Rating of the PANSS was performed by independent, experienced psychologists who were unaware of the results of other outcome measures. The CGI-SCH, GAF, DIEPSS, TIP-Sz, and FACT-Sz were rated by each attending physician, and subjective measures were completed by the participants. Body weight and vital signs (ie, body temperature, heart rate, and blood pressure) were monitored throughout the study. Routine blood work was performed every 12 weeks. 
For the sake of measurement-based treatment, we used the PANSS total score as a guide to decide subsequent treatments, as discussed below. Further, regular meetings were held to provide ongoing training and to ensure interrater reliability on the clinical scales. The ALGO group physicians were required to change psychopharmacological treatments based on the PANSS reduction rate. On the other hand, the psychopharmacological treatment of TAU physicians reflected a best case-by-case clinical judgment, irrespective of the PANSS scores.

The study assignment to ALGO or TAU was decided by the physicians, and not by the patients or the facilities. In other words, the study doctors were allocated to treat patients, either using the algorithm or on a TAU basis. All TAU physicians belonged to Ohizumi Hospital, which was intended so as to not masquerade TAU as ALGO (ie, TAU physicians under study try to intentionally modify their usual practices and imitate ALGO to reflect perceived study outcome desirability). All of the study data were monitored by the central research coordinator (MK) in order to maintain research integrity.

\section{Algorithm description}

There are some differences among antipsychotics; however, it is currently recommended to base the choice of antipsychotics on the adverse events profile rather than efficacy. Our psychopharmacological treatment algorithm is composed of four stages and is shown in Figure 1 (the rationale for our algorithm can be found in Table 1). This algorithm reflects currently available evidence in an effort to optimize antipsychotic treatment while avoiding irrational polypharmacy:

- Each treatment step lasted up to 12 weeks with antipsychotic monotherapy.

- In the first and second steps, when the posttreatment PANSS total score was above $70 \%$ of that at baseline (ie, the total PANSS score at the time of entry into the study), the patients were treated with the next antipsychotic.

- The dose of each antipsychotic was decided flexibly on an individual basis, with the basic clinical rule of beginning treatment at a low dose and titrating upwards to search for the lowest effective dose in the first 4 weeks, while monitoring tolerability and adverse effects.

- Antipsychotics included: olanzapine once daily, $\sim 20 \mathrm{mg} /$ day; risperidone once to three times daily, $\sim 12 \mathrm{mg} /$ day; quetiapine twice or three times daily, $\sim 750 \mathrm{mg} /$ day; perospirone (a Japanese serotonin-dopamine antagonist) once to three times daily, $48 \mathrm{mg} /$ day; blonanserin (another Japanese serotonin-dopamine antagonist) once to three times daily, $\sim 24 \mathrm{mg} /$ day; and aripiprazole once to two times daily, $\sim 30 \mathrm{mg} /$ day - all as recommended in their respective drug information sheets.

- From the third step onward, the PANSS threshold was reduced to $80 \%$ (as less resistant patients would have already responded before).

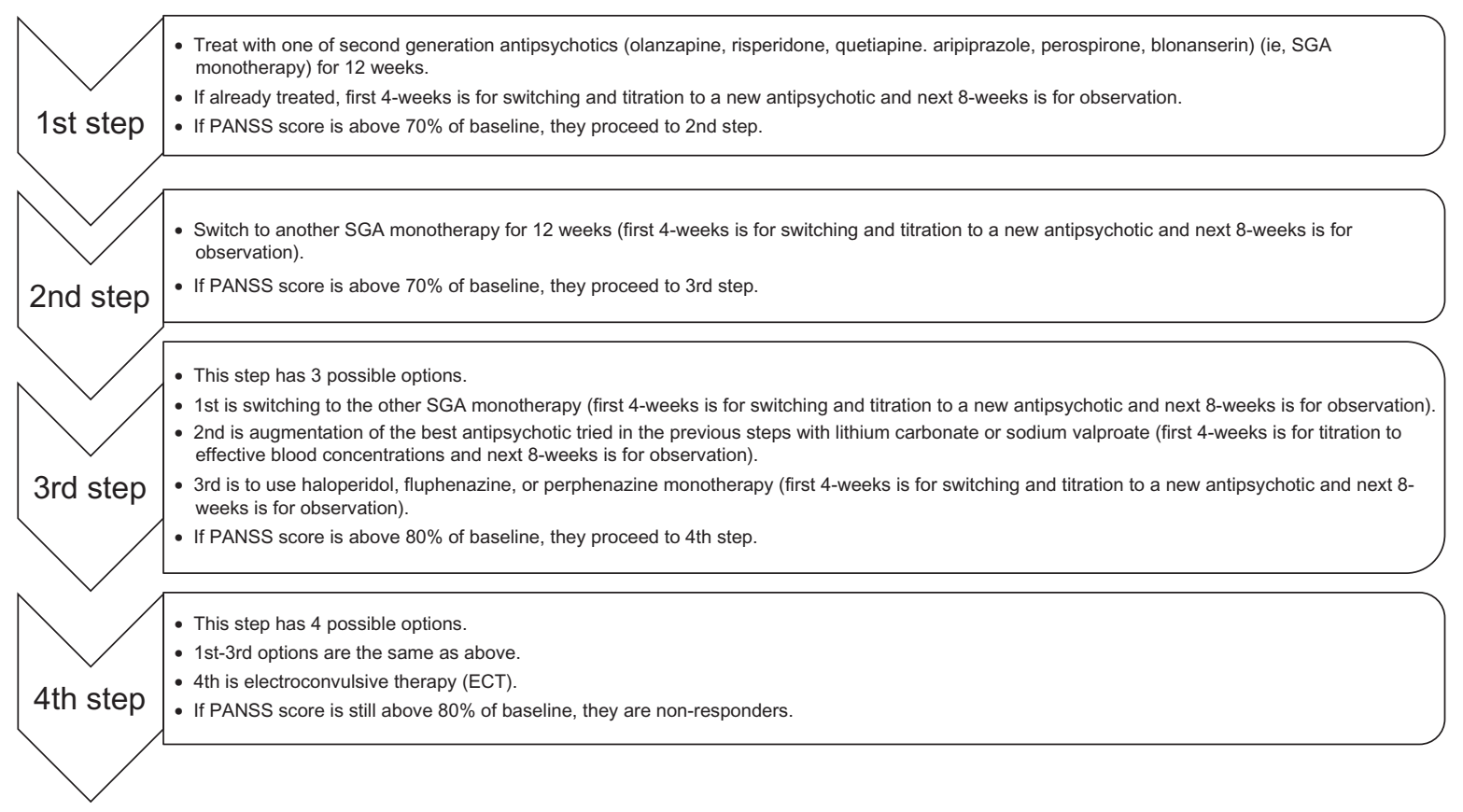

Figure I The treatment algorithm.

Abbreviation: PANSS, Positive and Negative Syndrome Scale. 
Table I Algorithm description

SGAs are getting more popular in reality and some guidelines support the use of SGAs over FGAs. ${ }^{17-19}$

If the first SGAs are not effective, there is some evidence that switching to another SGA can be effective. ${ }^{17,20,21}$

Even if SGAs are not effective, there may be room for FGAs in light of some equivocal differences (in efficacy, effectiveness, and adverse effects across antipsychotics) ${ }^{22-24}$ for clozapine (which was unavailable at the time of study in Japan).

There is some (albeit limited) evidence that concomitant treatment with lithium carbonate or valproate acid might be effective for difficult-to-treat schizophrenia. ${ }^{25,26}$

There is very little evidence for resistant patients who fail to respond to more than three different antipsychotics. No formal recommendation, including electroconvulsive therapy, can unequivocally be made at this treatment stage.

Our psychopharmacological treatment algorithm is based on the currently available evidence and expert opinions.

Note: This algorithm was developed by the third author TS in close collaboration with other contributors.

Abbreviations: SGA, second-generation antipsychotic; FGA, first-generation antipsychotic.

- Antipsychotics also included haloperidol or fluphenazine, once to three times daily $\sim 12 \mathrm{mg} /$ day; and perphenazine once to two times daily, $\sim 48 \mathrm{mg} /$ day.

- In the event that augmentation therapy with lithium carbonate or sodium valproate was selected, the dose was titrated upwards to effective blood concentrations (ie, lithium, 0.4 1.2 $\mathrm{mEq} / \mathrm{L}$; valproate, $50 \sim 125 \mathrm{ng} / \mathrm{mL}$ ).

- In all steps, the use of lorazepam $\sim 6 \mathrm{mg} / \mathrm{day}$ for anxiety/insomnia/agitation and/or biperiden $\sim 3 \mathrm{mg}$ /day for extrapyramidal symptoms was permitted only when clinically necessary.

- In the event that troublesome side effects argued against continuation of treatment, ALGO patients were allowed to go to the next step prematurely before the predetermined timelines at any treatment stages.

\section{Statistical analyses}

The differences between the ALGO and TAU groups in each rating scale were investigated. All analyses were conducted with last-observed-carried-forward principle, using the intention-to-treat population. Continuous variables were analyzed by Mann-Whitney's U-test for nonnormal data, and categorical variables were evaluated by Student's $t$-test. Study retention was compared using Kaplan-Meier survival analysis. A $P$-value of $<0.05$ was considered to indicate statistical significance (two-tailed).

\section{Results}

A total of 48 patients met the inclusion criteria for the study and provided informed consent. Six of these patients did not complete the baseline assessments, leaving 42 patients to be studied. The characteristics of the participants at baseline are shown in Table 2. There were 25 ALGO patients and 17 TAU patients. There were no significant differences in age, sex, duration of illness, previous number of admissions, or age at onset between the groups. Likewise, no significant differences were noted in the CGI-SCH, FACT-Sz, TIP-Sz, GAF, SWN-J, DIEPSS, and SF-36v2 scores. However, the baseline PANSS total score was significantly higher in the ALGO group than in TAU group (mean \pm standard deviation [SD]: ALGO, 106.9 \pm 20.0 and TAU, $92.2 \pm 18.3$; U-test $P=0.021)$. Therefore, changes in the PANSS total score were interpreted in terms of the percent reduction from baseline scores in order to take into account such imbalances in symptom severity between the groups.

Our clinical trial resulted in a high dropout rate of $64 \%$ in the ALGO group and $71 \%$ in the TAU group (Figure S1). The PANSS reduction rates in the two groups failed to show any significant differences at all times (at 24 weeks: ALGO mean $\pm \mathrm{SD}$ reduction rate, $0.1738 \pm 0.201$; TAU, $0.152 \pm 0.109$; U-test $P=0.672$; at 52 weeks: ALGO, $0.177 \pm 0.194$; TAU $0.187 \pm 0.157$; U-test $P=0.847$ ) (Table 3 and Figure 2).

Likewise, we could not find any evidence for significant differences in the ALGO group compared to the TAU group regarding secondary outcomes. For instance, the results of the CGI-SCH as well as the GAF and TIP-Sz corroborated the PANSS results and were nonsignificant (Table 3). Further, there were no significant differences in extrapyramidal symptoms as assessed by the DIEPSS, and only two cases (one in each group) needed an anti-Parkinson drug. One patient in the ALGO group responded to risperidone, but later showed dystonia, which was resolved by switching to quetiapine.

Chlorpromazine-equivalent antipsychotic dosage (mean $\pm \mathrm{SD}$ ) was $386.6 \pm 258.5 \mathrm{mg}$ for the ALGO group and $540.4 \pm 362.5 \mathrm{mg}$ for the TAU group at 24 weeks, which failed to reach statistical significance likely due to the limited number of patients, a wider variability in dosage requirements, and more attritions at later treatment weeks (Figure S2). There were no safety concerns for unexpected adverse reactions in both treatment groups throughout the study. 
Table 2 Baseline characteristics of ALGO and TAU groups

\begin{tabular}{|c|c|c|c|c|}
\hline & ALGO group & TAU group & Total & $P$-value \\
\hline Number of patients & 25 & 17 & 42 & \\
\hline Sex & & & & 0.116 \\
\hline Male & $15(60 \%)$ & $6(35 \%)$ & $21(50 \%)$ & \\
\hline Female & $10(40 \%)$ & II (65\%) & $21(50 \%)$ & \\
\hline Age (years) ${ }^{b}$ & $40.1(11.9)$ & $37.5(9.4)$ & $39.0(10.9)$ & 0.433 \\
\hline Education $\left(\right.$ years) ${ }^{b}$ & $12.7(1.7)$ & $13.6(1.7)$ & $13.2(1.7)$ & 0.270 \\
\hline Employment ${ }^{\mathrm{a}}$ & & & & 0.632 \\
\hline Full-time & $6(24 \%)$ & $4(24 \%)$ & $10(24 \%)$ & \\
\hline Part-time & $0(0 \%)$ & $2(12 \%)$ & $2(5 \%)$ & \\
\hline Unemployed & $19(76 \%)$ & II (64\%) & 30 (7।\%) & \\
\hline Subtype ${ }^{a}$ & & & & 0.139 \\
\hline Paranoid type & $24(96 \%)$ & 14 (82\%) & 38 (91\%) & \\
\hline Disorganized type & I (4\%) & $2(12 \%)$ & $3(7 \%)$ & \\
\hline Hebephrenic type & $0(0 \%)$ & I (6\%) & I (2\%) & \\
\hline Hospitalization ${ }^{\mathrm{a}}$ & & & & 0.174 \\
\hline Inpatient & $14(56 \%)$ & $13(76 \%)$ & 27 (64\%) & \\
\hline Involuntary admission & II (44\%) & $9(53 \%)$ & $20(48 \%)$ & \\
\hline Outpatient & II (44\%) & $4(24 \%)$ & $15(36 \%)$ & \\
\hline Age of onset (years) ${ }^{b}$ & $30.2(12.2)$ & $29.8(9.9)$ & $30.1(11.2)$ & 0.904 \\
\hline Duration of untreated psychosis (months) ${ }^{\mathrm{b}}$ & $18.0(32.0)$ & $13.7(19.2)$ & $16.6(27.6)$ & 0.589 \\
\hline Duration of illness (months) ${ }^{b}$ & $115.1(123.8)$ & $104.5(95.8)$ & $113.6(112.1)$ & 0.757 \\
\hline Number of admissions ${ }^{b}$ & $1.2(1.6)$ & $1.4(1.8)$ & $\mathrm{I} .3(1.7)$ & 0.755 \\
\hline PANSS total score ${ }^{c}$ & $106.9(20.0)$ & $92.2(18.3)$ & $101.0(20.4)$ & 0.021 \\
\hline Positive $^{c}$ & $27.6(5.9)$ & $23.0(6.3)$ & $25.8(6.4)$ & 0.038 \\
\hline Negative $^{c}$ & $25.2(4.8)$ & $21.9(6.3)$ & $23.9(5.6)$ & 0.070 \\
\hline General psychopathologyc & $54.0(11.7)$ & $47.3(8.9)$ & $51.3(11.1)$ & 0.053 \\
\hline $\mathrm{GAF}^{\mathrm{c}}$ & $43.6(15.4)$ & $47.0(20.0)$ & $45.0(17.3)$ & 0.452 \\
\hline CGI-SCH ${ }^{c}$ & $20.2(4.0)$ & $18.6(7.0)$ & $19.6(5.4)$ & 0.280 \\
\hline FACT-Sz ${ }^{c}$ & $44.8(16.2)$ & $50.8(20.5)$ & $47.2(18.1)$ & 0.315 \\
\hline TIP-Sz ${ }^{c}$ & $52.0(14.2)$ & $58.4(19.3)$ & $54.6(16.5)$ & 0.127 \\
\hline DIEPSS ${ }^{c}$ & $0.84(1.5)$ & $0.58(1.0)$ & $0.74(1.3)$ & 0.790 \\
\hline SWN-jc & 49.5 (16.9) & $55.4(22.8)$ & $51.1(19.5)$ & 0.434 \\
\hline DAI- $10^{c}$ & $2.1(4.8)$ & $0.06(4.5)$ & I.3 (4.7) & 0.162 \\
\hline$S F-36 v 2^{c}$ & $104.7(12.9)$ & $104.8(10.3)$ & 104.7 (II.8) & 0.868 \\
\hline $\begin{array}{l}\text { Antipsychotic dose (chlorpromazine } \\
\text { equivalent, } \mathrm{mg} / \text { day) }\end{array}$ & $416.2(3 \mid 8.7)$ & $318.4(215.9)$ & $378.7(289.3)$ & 0.313 \\
\hline
\end{tabular}

Notes: Data are represented as number (percentage) or mean (standard deviation) unless stated otherwise. ${ }^{\text {aPearson's }} \chi^{2}$ test; 'bindependent Student's $t$-test; ‘Mann-Whitney U-test.

Abbreviations: ALGO, algorithm-based treatment; TAU, treatment-as-usual; PANSS, Positive and Negative Syndrome Scale; GAF, Global Assessment of Functioning; CGISCH, Clinical Global Impression-Schizophrenia; FACT-Sz, Functional Assessment for Comprehensive Treatment of Schizophrenia; TIP-Sz, Targeted Inventory on Problems in Schizophrenia; DIEPSS, Drug Induced Extra-Pyramidal Symptoms Scale; SWN-J, Subjective Well-Being under Neuroleptics-Japanese; DAI-I0, Drug Attitude Inventory-I0; SF-36v2, Short-Form 36v2 Health Survey.

Looking at the treatment details (Figure S3), of the 25 ALGO cases entered, six responded, eleven prematurely dropped out, six deviated from the algorithm, and two completed the study without satisfying our response criteria. Alternatively, the response rate was $26.3 \%$ (5/19) in the first step, $33.0 \%(1 / 3)$ in the third step, but $0 \%$ for the rest of the steps. On the other hand, the response rate in the TAU group was $23.5 \%(4 / 17)$ according to the $\leq 70 \%$ threshold, and $41.2 \%$ (7/17) for the $80 \%$ cutoff in the PANSS. Nine patients dropped out in the midst of treatment, and two completed the algorithm but did not respond according to our PANSS criteria.
As for the antipsychotics selected in the ALGO group (Figure S4), risperidone, olanzapine, and aripiprazole were popular. It was found that treatments selected in the TAU group were very similar in quality to those in the ALGO group. In fact, psychopharmacological therapy was "completely" identical to our algorithm in six instances, and in four other cases, it conformed to our algorithm in that lorazepam was only replaced with other benzodiazepines (Figure S5). The average dose of antipsychotics was not significantly different between the groups (Table S1). A small number of patients treated with their respective antipsychotics did not 
Table 3 Changes in clinical scales for ALGO and TAU patients

\begin{tabular}{|c|c|c|c|c|c|c|c|c|c|}
\hline & \multicolumn{3}{|l|}{0 weeks } & \multicolumn{3}{|l|}{24 weeks } & \multicolumn{3}{|l|}{52 weeks } \\
\hline & ALGO & TAU & $P$-value & ALGO & TAU & $P$-value & ALGO & TAU & $P$-value \\
\hline \multicolumn{10}{|l|}{ PANSS } \\
\hline Positive & $27.6(5.9)$ & $23.0(6.3)$ & 0.038 & $21.4(7.0)$ & 19.1 (5.6) & 0.311 & $20.2(6.2)$ & I7.7 (5.7) & 0.464 \\
\hline Negative & $25.2(4.8)$ & $21.9(6.3)$ & 0.070 & $21.1(5.2)$ & I9.| (5.6) & 0.190 & $20.6(4.7)$ & $19.2(7.0)$ & 0.217 \\
\hline General & 54.0 (II.7) & $47.3(8.9)$ & 0.053 & $44.0(9.5)$ & 39.7 (8.4) & 0.093 & $42.0(8.2)$ & $37.9(10.1)$ & 0.259 \\
\hline \multicolumn{10}{|c|}{ psychopathology } \\
\hline Total & $106.9(20.0)$ & $92.2(18.3)$ & 0.021 & $86.6(19.5)$ & 77.9 (16.7) & 0.096 & $82.8(17.0)$ & $74.9(19.4)$ & 0.200 \\
\hline GAF & $43.6(15.4)$ & $47.0(20.0)$ & 0.452 & $59.8(12.6)$ & $59.7(14.5)$ & 0.815 & $59.5(12.9)$ & $60.5(15.0)$ & 0.501 \\
\hline CGI-SCH & $20.2(4.0)$ & $18.6(7.0)$ & 0.280 & $14.6(4.9)$ & $14.7(6.4)$ & 0.990 & $14.6(4.8)$ & $14.4(6.5)$ & 0.758 \\
\hline FACT-Sz & $44.8(16.2)$ & $50.8(20.5)$ & 0.315 & $63.2(12.1)$ & $61.3(17.2)$ & 0.680 & $59.8(17.2)$ & $61.9(17.4)$ & 0.796 \\
\hline TIP-Sz & $52.0(14.2)$ & $58.4(19.3)$ & 0.127 & $68.4(13.0)$ & $71.6(14.5)$ & 0.311 & $68.8(13.5)$ & $72.8(14.7)$ & 0.195 \\
\hline DIEPPS & $0.84(1.5)$ & $0.58(1.0)$ & 0.790 & $1.68(3.6)$ & I.47 (3.0) & 0.962 & $1.6(3.7)$ & I.I (5.7) & 0.817 \\
\hline SWN-J & 49.5 (16.9) & $55.4(22.8)$ & 0.434 & $48.4(17.6)$ & $59.1(22.1)$ & 0.093 & $50.3(17.4)$ & $62.0(19.8)$ & 0.051 \\
\hline DAI-10 & $2.1(4.8)$ & $0.06(4.5)$ & 0.162 & $1.0(4.8)$ & $2.1(5.5)$ & 0.508 & $0.8(4.9)$ & I.I2(5.7) & 0.948 \\
\hline SF-36v2 & $104.7(12.9)$ & $104.8(10.3)$ & 0.868 & $103.0(10.7)$ & $107.6(8.8)$ & 0.079 & $103.7(12.3)$ & $108.6(11.6)$ & 0.124 \\
\hline $\begin{array}{l}\text { Antipsychotic } \\
\text { dose (mg/day) }\end{array}$ & $416.2(318.7)$ & $318.4(215.9)$ & 0.313 & $386.6(258.5)$ & $540.4(362.5)$ & 0.143 & $44 I . I$ (308.5) & $475.7(345.6)$ & $0.74 I$ \\
\hline
\end{tabular}

Notes: Data are represented by the mean (standard deviation). ALGO group, $n=25$; TAU group, $n=17$. All statistical analyses were conducted by the Mann-Whitney U-test.

Abbreviations: ALGO, algorithm-based treatment; TAU, treatment-as-usual; PANSS, Positive and Negative Syndrome Scale; GAF, Global Assessment of Functioning; CGISCH, Clinical Global Impression-Schizophrenia; FACT-Sz, Functional Assessment for Comprehensive Treatment of Schizophrenia; TIP-Sz, Targeted Inventory on Problems in Schizophrenia; DIEPSS, Drug Induced Extra-Pyramidal Symptoms Scale; SWN-J, Subjective Well-Being under Neuroleptics-Japanese; DAI-I0, Drug Attitude Inventory-I0; SF-36v2, Short-Form 36v2 Health Survey.

allow meaningful statistics to shed light on differential effects among antipsychotics.

\section{Discussion}

This study represents one of very few studies to evaluate the usefulness and feasibility of measurement and algorithmbased, sequential pharmacotherapy with antipsychotics for patients with schizophrenia in real-world clinical settings. ${ }^{20}$
The ALGO group compared well with the TAU group, although patients in this study presented with relatively severe psychopathology in that the PANSS total score at baseline was high at 106.9 and 92.2, respectively, for each group. Furthermore, a majority of patients were inpatients in this study, which is in contrast to previous studies that solely included outpatients. ${ }^{17} \mathrm{TAU}$ treatment that was intended to represent a best case-by-case clinical judgment was akin in

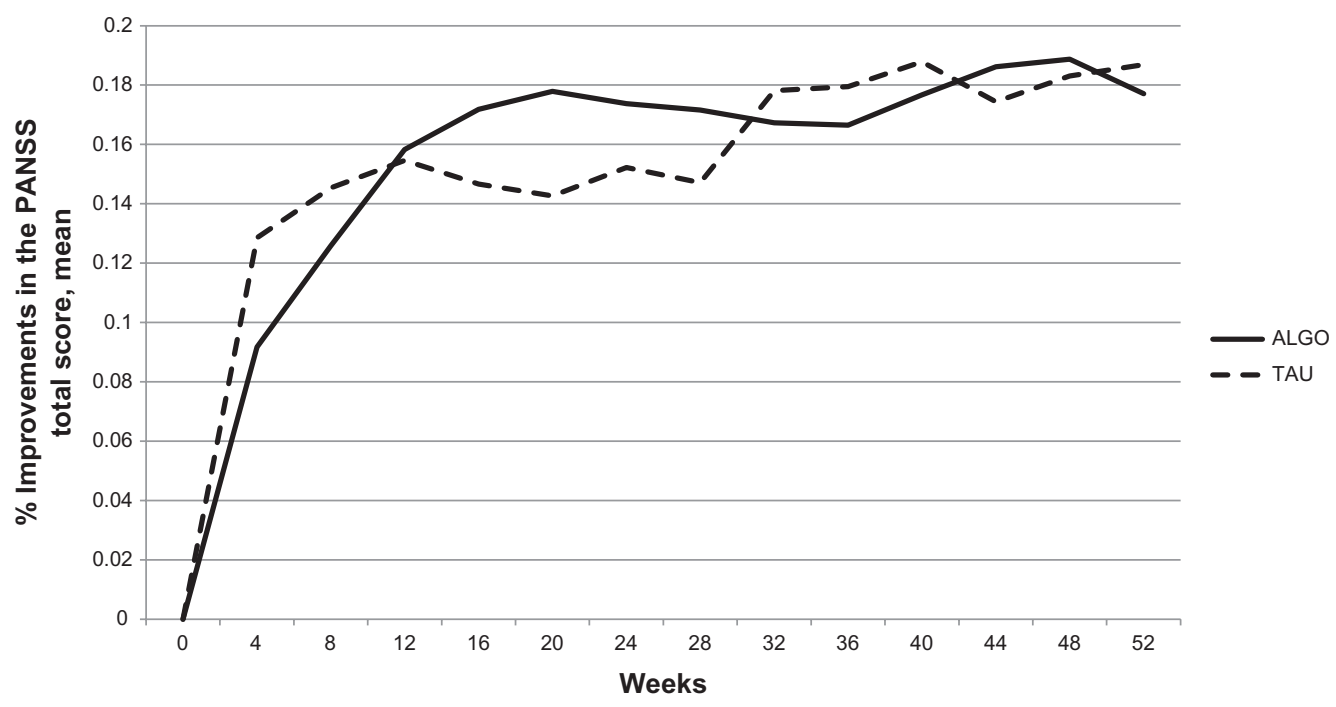

Figure 2 Improvements in the ALGO and TAU groups.

Note: The PANSS total scores = the baseline scores $\times(I-$ the numbers on the $Y$-axis at each time point).

Abbreviations: ALGO, algorithm-based treatment; TAU, treatment-as-usual; PANSS, Positive and Negative Syndrome Scale. 
quality to the ALGO in this study (ie, no differences emerged between the treatment groups), which significantly complicated the interpretation of our results.

We failed to find an advantage in terms of the primary outcome - the PANSS total score - as well as in a variety of relevant secondary outcome measures that encompassed global functioning (GAF and FACT-Sz), extrapyramidal symptoms (DIEPSS), problems and overall severity (TIP-Sz, CGI-SCH), subjective well-being (SWN-J), quality of life (SF-36v2), and drug preferences (DAI-10). ${ }^{27}$ We could not find any significant superiority of ALGO compared to TAU, but our data suggest that ALGO might manage more severe illness with a smaller amount of antipsychotics. Our work indicates that testing ALGO for schizophrenia is a feasible clinical goal.

In this clinical trial, patients in the ALGO and TAU groups were not randomly assigned or matched. Treatment allocation to the ALGO or the TAU group was decided according to the physician, and not according to the patients or the facilities. We have made an effort to avoid "treatment contamination" by recruiting all TAU patients from a single institution, but this study suffered from a high dropout rate. This may be related to the fact that the patients appeared to have rather symptomatic illness, especially considering the baseline PANSS score, although no formal assessments were made for treatment resistance. ${ }^{28}$ Nevertheless, $64.3 \%$ of patients were inpatients, with involuntary admission accounting for $74.0 \%$ of patients. In addition, this was a 1-year study and many of these patients with suboptimal insight and substantial psychopathology were not successful for continued treatment. Other patients were simply inaccessible due to location. This is relevant in Japan, since there are patients who cannot choose which hospitals to be admitted to in case of compulsory hospitalization where a risk of harming oneself or others is noted. In such cases, patients frequently go back to their hometown, making an establishment of catchment area extremely complicated in a crowded city, while this is highly desirable for longer-term studies. Location issues accounted for six dropouts in each group.

Miller et $\mathrm{al}^{2}$ argued that algorithm-based intervention encouraged clinicians to be more aggressive in treating residual symptoms, which is compatible with our clinical trial. In the TAU group, only seven cases switched their antipsychotics in the middle of treatment. On the other hand, patients in the ALGO group experienced more antipsychotic trials, which is in part due to the fact that patients failing to meet the predetermined cutoff points should uniformly have gone to the next step in the study protocol. One important consideration is to take into account the stage of the illness, since first-episode patients are known to be responsive to antipsychotic medications, although they are sensitive as well $;{ }^{29}$ in addition, the greater number of antipsychotics that patients failed to respond to, the less likely they were to be responsive to another. ${ }^{30}$ How aggressive goal setting could be or how realistic it should be among patients with schizophrenia in general, or with patients exhibiting some specific characteristics (eg, treatment-resistant schizophrenia [TRS]) would be a matter for further investigation.

While this study represents one of a few endeavors of measurement-based treatment and ALGO in schizophrenia, ${ }^{2,3}$ and while it tries to capture important domains of the illness with the use of independent raters for the main outcome of interest, there are a number of limitations to be noted. First, the number of participants was rather small, and there was a high premature dropout rate, which limits the representativeness of the sample and leaves a possibility of a type- 2 error. We stratified patients by physicians and cannot know for sure how the study design may have potentially biased the results; however, a lack of patientlevel randomization, as is typical for clinical trials, is an obvious limitation. Potential individual differences in the effectiveness and safety of antipsychotics could not be inferred from this small study. ${ }^{31}$ Second, the availability of newer antipsychotics was limited. While perispirone and blonanserin were included as atypical agents, amisulpiride, ziprasidone, paliperidone, asenapine, lurasidone, iloperidone, and clozapine were not evaluated. This is especially true for clozapine, which shows superiority for TRS, ${ }^{32}$ but this drug was unavailable in Japan at the time of the study. While clozapine has been regarded as the third medication, its position needs to be further examined in the treatment algorithm of schizophrenia. ${ }^{33}$ Third, treatment allocation was guided by the physicians and not by the patients or the facilities. As such, physicians' prescribing tendencies could not be controlled for. This issue has affected the results in a significant manner, as discussed. Fourth, our patients exhibited relatively severe illness, and the baseline PANSS score was higher in the ALGO group than in the TAU group. Although a higher score may indicate more room for improvement, it may contrarily be an indication of more difficult-to-treat conditions. Fifth, a concept of ALGO treatment may sound the opposite to individually-tailored treatment. ${ }^{34}$ We believe both perspectives are important. Finally, there has been no unequivocal consensus on what constitutes reasonable assessment measures, but the PANSS has been regarded as a "standard." 35 In this context, we used the PANSS as the 
primary outcome measure. Although a formal sample size calculation would have been ideal, it was not feasible as a lack of good data on this topic prevented us to perform sample size estimation. In fact, the past few studies have failed to find an effect size that was large enough to allow for a realistic number of patients for inclusion.

A fair interpretation of the results of this study appears to be that ALGO compares well to TAU, rendering ALGO a rational approach and a topic of further scrutiny. In TAU, ten patients received treatment, which was - in essence - the same with our algorithm (see Results). Altogether, the treatment results of these 35 patients ( 25 from the ALGO group and ten from the TAU group), which are shown in Table S2 and are equivalent to a $27 \%$ reduction of the total PANSS score over the treatment period, could serve as an indicator of the overall usefulness of our algorithm in these symptomatic patients (see Suzuki ${ }^{36}$ for past evidence on the PANSS/BPRS scores in TRS patients).

\section{Conclusion}

To conclude, the results showed that algorithm-based antipsychotic treatment compared well to TAU, but the results need to be interpreted in a context of limitations in the study, especially nearly indistinguishable psychopharmacotherapy between ALGO and TAU. Further investigations are clearly warranted on this highly pertinent topic.

\section{Acknowledgments}

The authors are grateful to Drs Motoyasu Ishii, Yasushi Moriyama, Norifusa Sawada, Kaoru Shimizu, Masayuki Tomita, Shinya Kojima, and Leina Tatsumi at Ohizumi Hospital for participating in this clinical trial as Treatment-As-Usual doctors. The authors are also grateful to Ms Fumie Saito at Keio University Hospital.

\section{Disclosure}

Role of funding source: This study has received funding from a Health and Labour Sciences Research Grant for Research on Psychiatric and Neurological Diseases and Mental Health (H20-KOKORO-003) from the Japanese Ministry of Health, Labour and Welfare to the last author MK. The funding source had no role in study design, data collection, data analysis, data interpretation, or writing of the report.

Dr Hirano has received manuscript or speaker's fees from Dainippon Sumitomo Pharma, Eli Lilly, Meiji Pharma, and GlaxoSmith Kline Janssen Pharma.

Dr Watanabe has received manuscript or speaker's fees from Astellas Pharma, Dainippon Sumitomo Pharma, Eli
Lilly Japan, GlaxoSmithKline, Janssen Pharmaceutical, Meiji Seika Pharma, Mitsubishi Tanabe Pharma, Mochida Pharmaceutical, Otsuka Pharmaceutical, Pfizer Japan, Shionogi, Yoshitomiyakuhin, and grants from Dainippon Sumitomo Pharma, GlaxoSmithKline, Meiji Seika Pharma, Mitsubishi Tanabe Pharma, Mochida Pharmaceutical, Otsuka Pharmaceutical, and Pfizer Japan.

Dr Suzuki has received manuscript or speaker's fees from Dainippon Sumitomo Pharma, Eli Lilly, Astellas Pharma, Novartis Pharma, and Meiji Seika Pharma.

Dr Uchida has received grants from Pfizer and DainipponSumitomo Pharma, and speaker's honoraria from Otsuka Pharmaceutical, Eli Lilly, Novartis Pharma, Shionogi, GlaxoSmithKline, Yoshitomi Yakuhin, Dainippon-Sumitomo Pharma, and Janssen Pharmaceutical.

Dr Nagasawa has received speaker's fees from Dainippon Sumitomo Pharma and Otsuka Pharmaceutical.

Dr Hara has received speaker's fees from Otsuka Pharmaceutical, Pfizer Japan, GlaxoSmithKline, Yoshitomi Yakuhin, Takeda Pharmaceutical.

Dr Inagaki has received grants from the Japan Pharmaceutical Manufacturer Association and Janssen Pharma, and manuscript or speaker's fees from Janssen Pharma, Astellas Pharma, Novartis Pharma, Eli Lilly Japan, Otsuka Pharmaceutical, Meiji Seika Pharma, and MSD Japan.

Dr Motohashi has received grants from Astellas Pharma, GlaxoSmithKline, Otsuka Pharmaceutical, Pfizer Japan, Sanofi-Aventis, and Yoshitomi Yakuhin.

Dr Mimura has received grants, consultant fees, and/ or speaker's honoraria from Asahi Kasei, Astellas, Daiichi Sankyo, Dainippon Sumitomo, Eisai, Eli Lilly, GlaxoSmithKline, Janssen, Meiji, Mochida, Novartis, Otsuka, Pfizer, Shionogi, and Yoshitomi.

The other authors do not have any conflicts of interests in relation to this manuscript. The authors report no other conflicts of interest in this work.

\section{References}

1. Correll CU, Kishimoto T, Nielsen J, Kane JM. Quantifying clinical relevance in the treatment of schizophrenia. Clin Ther. 2011;33(12): B16-B39.

2. Miller AL, Crismon ML, Rush AJ, et al. The Texas medication algorithm project: clinical results for schizophrenia. Schizophr Bull. 2004;30(3):627-647.

3. Janssen B, Ludwig S, Eustermann H, et al. Improving outpatient treatment in schizophrenia: effects of computerized guideline implementation - results of a multicenter-study within the German research network on schizophrenia. Eur Arch Psychiatry Clin Neurosci. 2010;260(1):51-57.

4. Trivedi MH, Rush AJ, Crismon ML, et al. Clinical results for patients with major depressive disorder in the Texas Medication Algorithm Project. Arch Gen Psychiatry. 2004;61(7):669-680. 
5. Suzuki T, Uchida H, Watanabe K, Yagi G, Kashima H. A clinical case series of switching from antipsychotic polypharmacy to monotherapy with a second-generation agent on patients with chronic schizophrenia. Prog Neuropsychopharmacol Biol Psychiatry. 2004;28(2):361-369.

6. Suzuki T, Uchida H, Tanaka KF, et al. Reducing the dose of antipsychotic medications for those who had been treated with high-dose antipsychotic polypharmacy: an open study of dose reduction for chronic schizophrenia. Int Clin Psychopharmacol. 2003;18(6):323-329.

7. Hasan A, Falkai P, Wobrock T, et al; for World Federation of Societies of Biological Psychiatry (WFSBP) Task Force on Treatment Guidelines for Schizophrenia. World Federation of Societies of Biological Psychiatry (WFSBP) Guidelines for Biological Treatment of Schizophrenia, part 1: update 2012 on the acute treatment of schizophrenia and the management of treatment resistance. World J Biol Psychiatry. 2012;13(5):318-378.

8. American Psychiatric Association. Diagnostic and Statistical Manual of Mental Disorders. 4th ed. Arlington, VA: American Psychiatric Association; 1994.

9. Haro JM, Kamath SA, Ochoa S, et al; for SOHO Study Group. The Clinical Global Impression-Schizophrenia scale: a simple instrument to measure the diversity of symptoms present in schizophrenia. Acta Psychiatr Scand Suppl. 2003;(416):16-23.

10. Inagaki A, Inada T. [Dose equivalence of psychotropic drugs. Part XXII: dose equivalence of depot antipsychotics III: risperidone longacting injection]. Japanese Journal of Clinical Psychopharmacology. 2010;13:1349-1353. Japanese.

11. Kay SR, Fiszbein A, Opler LA. The positive and negative syndrome scale (PANSS) for schizophrenia. Schizophr Bull. 1987;13(2): 261-276.

12. Inada T, Yagi G, Miura S. Extrapyramidal symptom profiles in Japanese patients with schizophrenia treated with olanzapine or haloperidol. Schizophr Res. 2002;57(2-3):227-238.

13. Suzuki T, Uchida H, Nomura K, et al. Novel rating scales for schizophrenia - Targeted Inventory on Problems in Schizophrenia (TIP-Sz) and Functional Assessment for Comprehensive Treatment of Schizophrenia (FACT-Sz). Schizophr Res. 2008;106(2-3):328-336.

14. Ware JE, Kosinski M, Dewey JE. How to Score Version Two of the SF-36 Health Survey. Lincoln, RI: QualityMetric, Incorporated, 2000

15. Naber D, Moritz S, Lambert M, et al. Improvement of schizophrenic patients' subjective well-being under atypical antipsychotic drugs. Schizophr Res. 2001;50(1-2):79-88.

16. Hogan TP, Awad AG, Eastwood R. A self-report scale predictive of drug compliance in schizophrenics: reliability and discriminative validity. Psychol Med. 1983;13(1):177-183.

17. Moore TA, Buchanan RW, Buckley PF, et al. The Texas Medication Algorithm Project antipsychotic algorithm for schizophrenia: 2006 update. J Clin Psychiatry. 2007;68(11):1751-1762.

18. Lehman AF, Kreyenbuhl J, Buchanan RW, et al. The Schizophrenia Patient Outcomes Research Team (PORT): updated treatment recommendations 2003. Schizophrenia Bull. 2004;30(2):193-217.

19. Weiden PJ. Discontinuing and switching antipsychotic medications: understanding the CATIE schizophrenia trial. J Clin Psychiatry. 2007;68 Suppl 1:12-19.

20. Suzuki T, Uchida H, Watanabe K, et al. How effective is it to sequentially switch among Olanzapine, Quetiapine and Risperidone? - A randomized, open-label study of algorithm-based antipsychotic treatment to patients with symptomatic schizophrenia in the real-world clinical setting. Psychopharmacology. 2007;195(2):285-295.
21. Takahashi H, Kamata M, Yoshida K, Ishigooka J, Higuchi H. Switching to olanzapine after unsuccessful treatment with risperidone during the first episode of schizophrenia: an open-label trial. J Clin Psychiatry. 2006;67(10):1577-1582.

22. Lieberman JA, Stroup TS, McEvoy JP, et al; for Clinical Antipsychotic Trials of Intervention Effectiveness (CATIE) Investigators. Effectiveness of antipsychotic drugs in patients with chronic schizophrenia. New Engl J Med. 2005;353(12):1209-1223.

23. Jones PB, Barnes TR, Davies L, et al. Randomized controlled trial of the effect on Quality of Life of second- vs first-generation antipsychotic drugs in schizophrenia: Cost Utility of the Latest Antipsychotic Drugs in Schizophrenia Study (CUtLASS). Arch Gen Psychiatry. 2006;63(10):1079-1087.

24. National Institute for Health and Care Excellence. Nice clinical guidelines Schizophrenia (update) (CG82). Core interventions in the treatment and management of schizophrenia in primary and secondary care (update). NICE 2009. Available from: http://publications.nice.org. uk/schizophrenia-cg82. Accessed 01 Jan 2013.

25. Leucht S, McGrath J, Kissling W. Lithium for schizophrenia. Cochrane Database Syst Rev. 2003;(3):CD003834.

26. Suzuki T, Uchida H, Takeuchi H, et al. Augmentation of atypical antipsychotics with valproic acid. An open-label study for most difficult patients with schizophrenia. Hum Psychopharmacol. 2009;24(8):628-638.

27. Remington G, Foussias G, Agid O. Progress in defining optimal treatment outcome in schizophrenia. CNS Drugs. 2010;24(1):9-20.

28. Suzuki T, Remington G, Mulsant BH, et al. Defining treatmentresistant schizophrenia and response to antipsychotics: a review and recommendation. Psychiatry Res. 2012;197(1-2):1-6.

29. Fleischhacker WW, Keet IP, Kahn RS; for EUFEST Steering Committee. The European First Episode Schizophrenia Trial (EUFEST): rationale and design of the trial. Schizophr Res. 2005;78(2-3):147-156.

30. Agid O, Arenovich T, Sajeev G, et al. An algorithm-based approach to first-episode schizophrenia: response rates over 3 prospective antipsychotic trials with a retrospective data analysis. J Clin Psychiatry. 2011;72(11):1439-1444.

31. Leucht S, Corves C, Arbter D, Engel RR, Li C, Davis JM. Second-generation versus first-generation antipsychotic drugs for schizophrenia: a meta-analysis. Lancet. 2009;373(9657):31-41.

32. Kane J, Honigfeld G, Singer J, Meltzer H. Clozapine for the treatment-resistant schizophrenic. A double-blind comparison with chlorpromazine. Arch Gen Psychiatry. 1988;45(9):789-796.

33. Agid O, Foussias G, Singh S, Remington G. Where to position clozapine: re-examining the evidence. Can J Psychiatry. 2010;55(10):677-684.

34. Uchida H, Mamo DC, Pollock BG, et al. Predicting plasma concentration of risperidone associated with dosage change: a population pharmacokinetic study. Ther Drug Monit. 2012;34(2):182-187.

35. Suzuki T. Which rating scales are regarded as 'the standard' in clinical trials for schizophrenia? A critical review. Psychopharmacol Bull. 2011;44(1):18-31.

36. Suzuki T, Remington G, Arenovich T, et al. Time course of improvement with antipsychotic medication in treatment-resistant schizophrenia. Br J Psychiatry. 2011;199(4):275-280. 


\section{Supplementary materials}

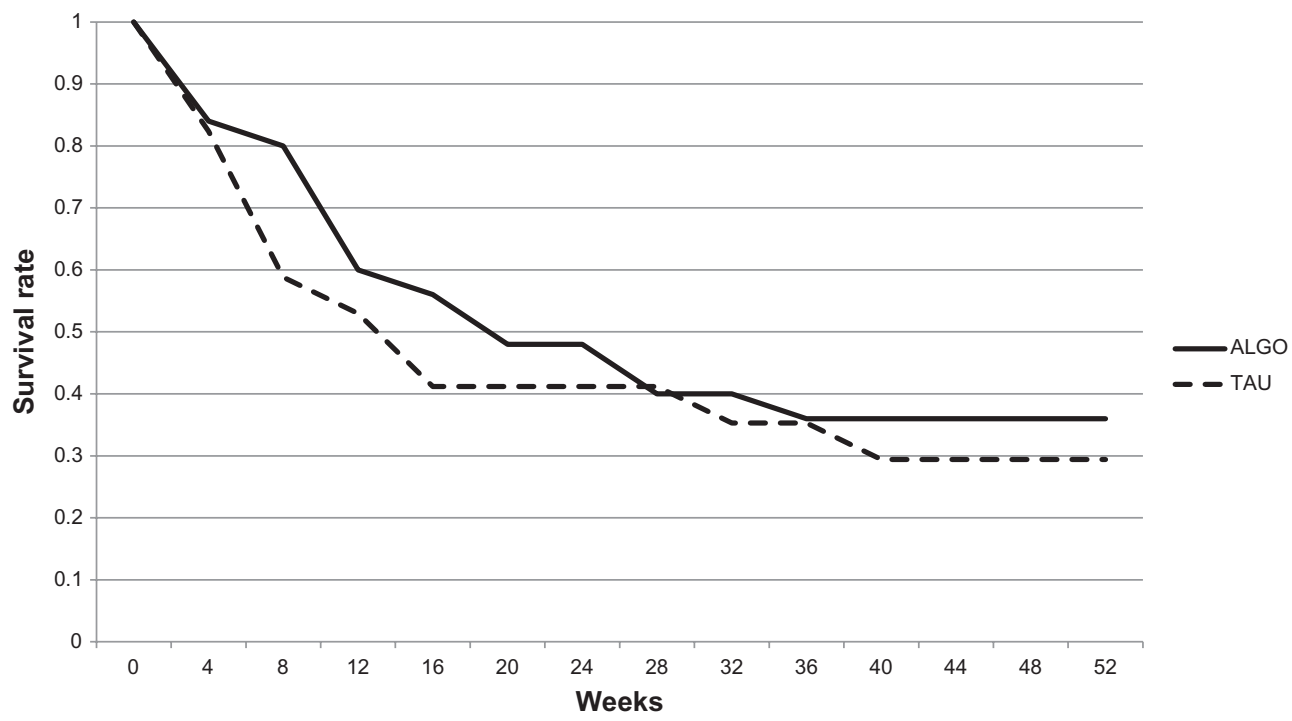

Figure SI Study retention of ALGO and TAU groups.

Abbreviations: ALGO, algorithm-based treatment; TAU, treatment-as-usual.

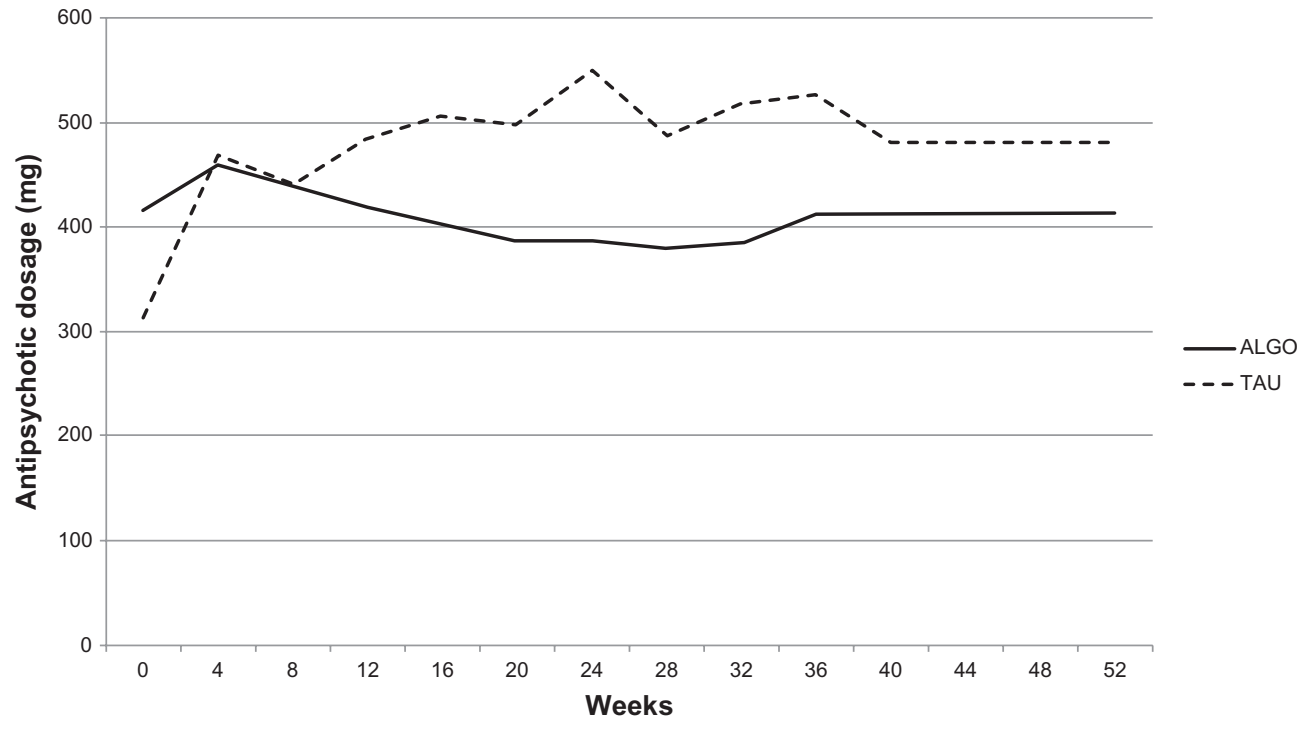

Figure S2 Mean chlorpromazine-equivalent dosage.

Abbreviations: ALGO, algorithm-based treatment; TAU, treatment-as-usual. 


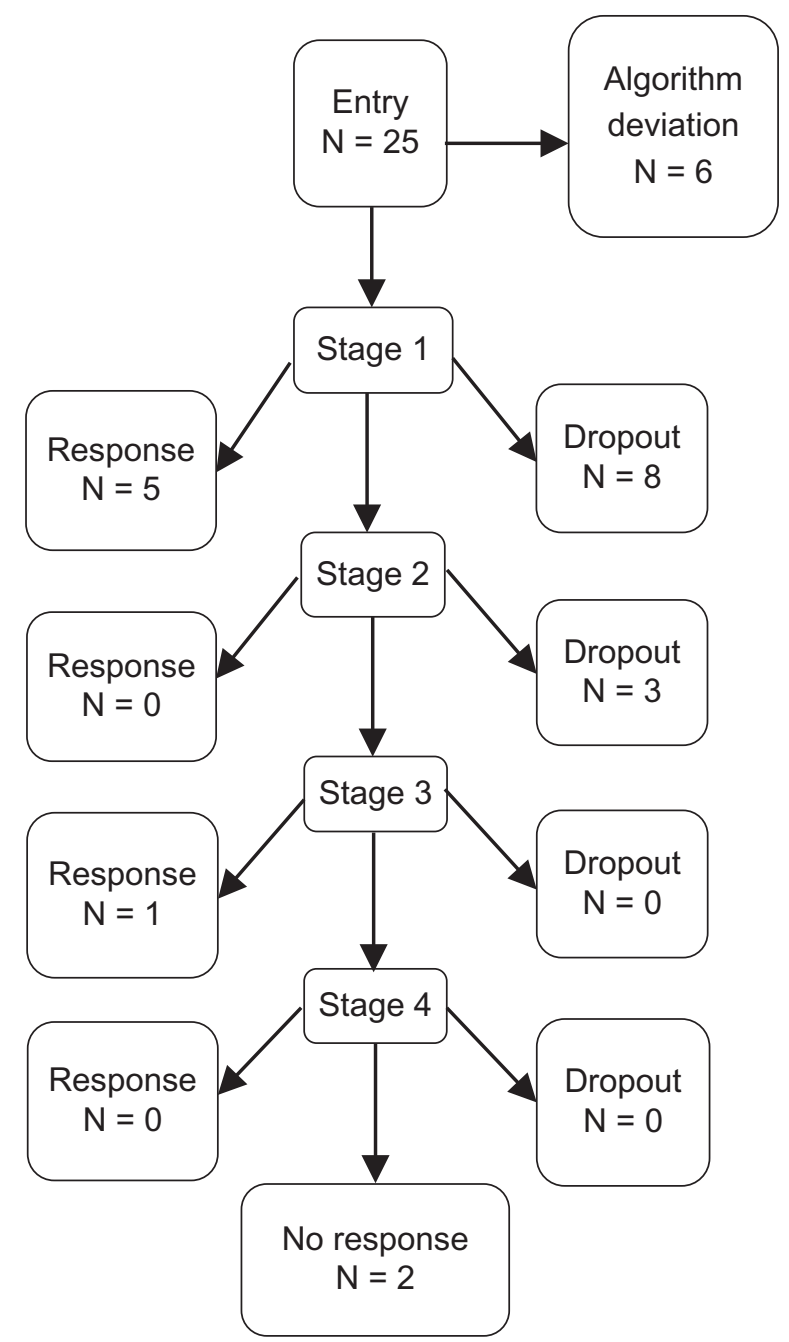

Figure S3 Outcome of ALGO.

Abbreviations: ALGO, algorithm-based treatment; N, number.

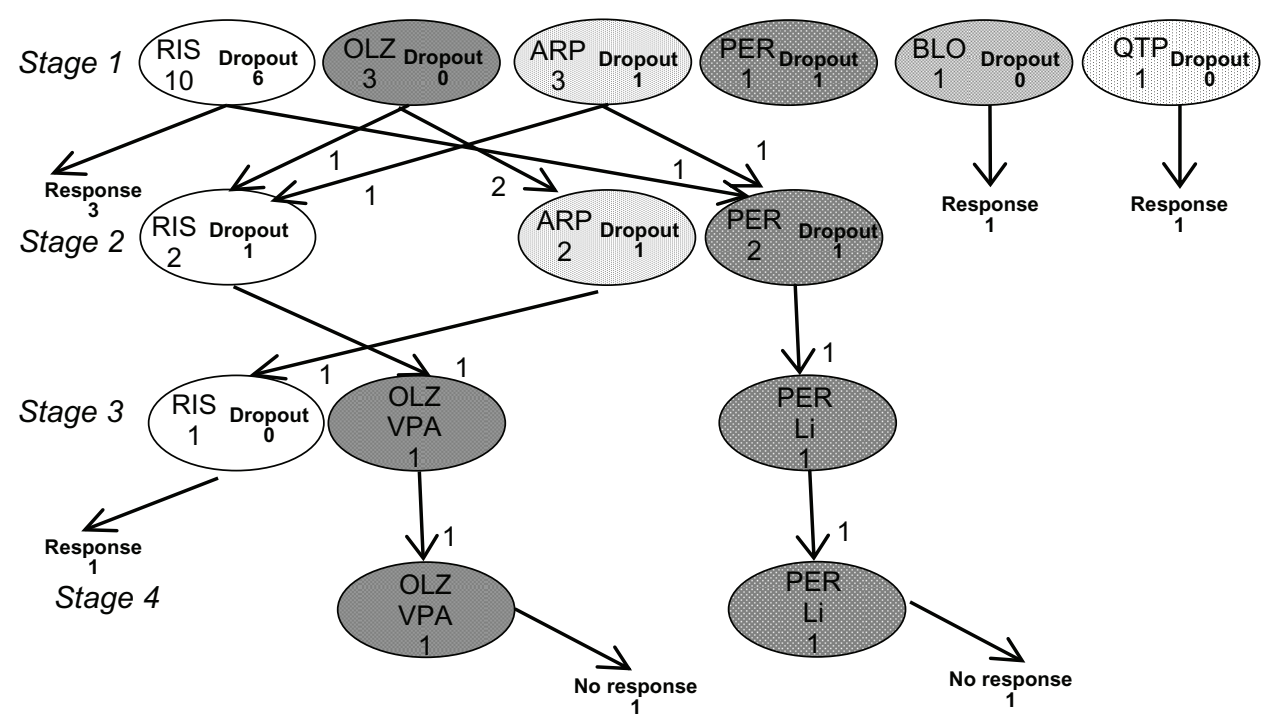

Figure S4 Outcome and antipsychotic choice in the ALGO group.

Abbreviations: ALGO, algorithm-based treatment; RIS, risperidone; OLZ, olanzapine; ARP, aripiprazole; PER, perospirone; BLO, blonanserin; QTP, quetiapine; VPA, valproate acid; Li, lithium carbonate. 


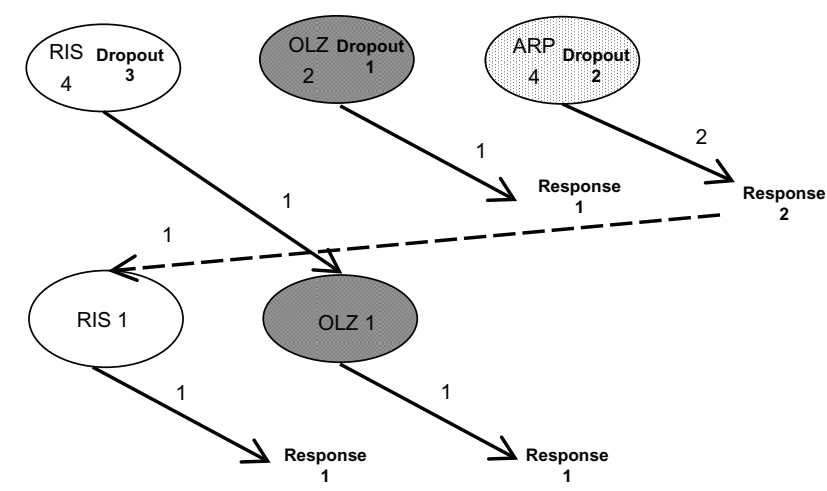

Figure S5 Outcome and antipsychotic choice in TAU patients, which "completely" and "nearly completely" conformed to our algorithm.

Notes: Those receiving treatment that "nearly completely" conformed to our algorithm used another single benzodiazepine than lorazepam. One patient showed severe akathisia with aripiprazole, which was resolved by a switch to risperidone.

Abbreviations: TAU, treatment-as-usual; RIS, risperidone; OLZ, olanzapine; ARP, aripiprazole.

Table SI The average dosage of SGAs in ALGO and TAU patients

\begin{tabular}{llll}
\hline & ALGO group & TAU group & P-value \\
\hline Risperidone & $5.0(3.6)$ & $4.0(2.0)$ & 0.119 \\
Olanzapine & $11.5(4.4)$ & $10.2(7.0)$ & 0.393 \\
Aripiprazole & $14.1(8.9)$ & $16.0(6.0)$ & 0.748 \\
Perospirone & $29.1(11.5)$ & - & \\
Quetiapine & $510.8(175.4)$ & - & \\
Blonanserin & $12.2(4.8)$ & - & \\
Valproate acid & $709.1(207.1)$ & - & \\
Lithium carbonate & $657.1(181.3)$ & - &
\end{tabular}

Notes: Data are the mean (standard deviation). Analysis was conducted by independent student's $t$-test.

Abbreviations: SGA, second-generation antipsychotics; ALGO, algorithm-based treatment; TAU, treatment-as-usual.
Table S2 Treatment results of 35 patients ( 25 from ALGO and ten from TAU who received "nearly completely" or "completely" equal treatment with our algorithm)

\begin{tabular}{|c|c|c|c|}
\hline & 0 weeks & 24 weeks & 52 weeks \\
\hline \multicolumn{4}{|l|}{ PANSS } \\
\hline Positive & $25.8(6.6)$ & $20.3(6.7)$ & $20.1(7.1)$ \\
\hline Negative & $24.1(4.8)$ & $20.4(4.9)$ & $20.0(4.9)$ \\
\hline $\begin{array}{l}\text { General } \\
\text { psychopatho }\end{array}$ & $52.1(10.6)$ & $42.9(9.3)$ & $42.3(9.9)$ \\
\hline Total & $102.0(19.2)$ & 83.6 (18.7) & $82.4(20.1)$ \\
\hline GAF & $46.9(17.2)$ & $6 \mathrm{I.3}(13.1)$ & $61.4(13.5)$ \\
\hline CGI-SCH & $18.9(5.0)$ & I4.0 (5.I) & $13.9(5.1)$ \\
\hline FACT-Sz & $49.7(17.0)$ & $64.7(12.8)$ & $62.2(16.8)$ \\
\hline TIP-Sz & $56.4(15.4)$ & $70.9(13.1)$ & $71.4(13.6)$ \\
\hline DIEPPS & $0.74(\mathrm{I} .4)$ & I.5I (3.4) & I.5 (3.4) \\
\hline SWN-J & $49.8(19.6)$ & $5 \mathrm{I} .0(20.8)$ & $52.7(19.5)$ \\
\hline DAI-I0 & I.I (4.4) & I.5 (4.6) & I.I (4.6) \\
\hline SF-36v2 & I04.I (II.5) & $104.4(9.6)$ & $105.5(11.2)$ \\
\hline $\begin{array}{l}\text { Antipsychotic } \\
\text { dose (mg/day) }\end{array}$ & $372.3(304.8)$ & $399.6(256.6)$ & $420.8(293.1)$ \\
\hline
\end{tabular}

Note: Data are the mean (standard deviation).

Abbreviations: ALGO, algorithm-based treatment; TAU, treatment-as-usual; PANSS, Positive and Negative Symptom Scale; GAF, Global Assessment of Functioning; CGI-SCH, Clinical Global Impression-Schizophrenia; FACT-Sz, Functional Assessment for Comprehensive Treatment of Schizophrenia; TIP-Sz, Targeted Inventory on Problems in Schizophrenia; DIEPSS, Drug Induced ExtraPyramidal Symptoms Scale; SWN-J, Subjective Well-Being under NeurolepticsJapanese; DAI-10, Drug Attitude Inventory-10; SF-36v2, Short-Form 36 v2 Health Survey.
Neuropsychiatric Disease and Treatment

\section{Publish your work in this journal}

Neuropsychiatric Disease and Treatment is an international, peerreviewed journal of clinical therapeutics and pharmacology focusing on concise rapid reporting of clinical or pre-clinical studies on a range of neuropsychiatric and neurological disorders. This journal is indexed on PubMed Central, the 'PsycINFO' database and CAS.

\section{Dovepress}

The manuscript management system is completely online and includes a very quick and fair peer-review system, which is all easy to use. Visit http://www.dovepress.com/testimonials.php to read real quotes from published authors. 